\title{
Effectiveness of Couple Therapy based on Imago Relationship Therapy: Its Impact on Couple Burnout
}

\author{
Davood Nazarpour ${ }^{1}$, Kianoush Zahrakar ${ }^{2}$, Seyed Mostafa Pouryahya ${ }^{3}$, Reza Davarniya ${ }^{2 *}$ \\ ${ }^{1}$ Department of Counseling, Allameh Tabataba'i University, Tehran, Iran \\ ${ }^{2}$ Department of Counseling, Kharazmi University, Tehran, Iran \\ ${ }^{3}$ Department of Counseling, Science and Research Branch, Islamic Azad University, Tehran, Iran
}

\section{ABSTRACT}

Introduction: The imago relationship approach is a theory on dating marriage and the process of marital conflicts treatment. Based on the main idea of this approach, choosing the spouse is done based on a mental image from ideal parents which has been formed in childhood, and compatibility with spouse is also reliant on this early image. The present study was designed to investigate the effectiveness of couple therapy based on imago relationship therapy on reducing couple burnout. Materials and Methods: The present research was a semi-empirical s tudy, which used a pretest-posttest with control group design. The statistical population of the present study included all the couples with marital conflicts referring to Rastin counseling and psychological services center in Gorgan, Iran, during the second quarter of 2018. The sample included 20 couples who were selected by available sampling method and were assigned into two experiment and control groups (10 couples per group) through ransom assignment method. The assessment of the subjects was conducted in pretest and posttest stages by using Pines' marital burnout scale (1996). Eight sessions of group couple therapy based on imago relationship therapy were held for the experiment group while the control group received no intervention. Results: The mean and standard deviation of the scores of couple burnout among the subjects of the experiment group in pretest and posttest stages equaled $81 \pm 21.97$ and $68.05 \pm 15.66$, respectively. The mean and standard deviation of the scores of couple burnout in the subjects of the control group equaled $80.95 \pm 16.79$ and $82.50 \pm 14.31$ in pre- and post-intervention, respectively. Results of the covariance analysis suggested that couple burnout in the couples of the experiment group has significantly decreased after receiving the imago relationship therapy intervention. Conclusion: Couple therapy based on imago relationship therapy enables the couples to understand one another, and experiencing this feeling can lead to increased happiness, intimacy, and empathy in couples and, therefore, reduce couple burnout.

\section{Key words:}

1. Couples Therapy

2. Burnout, Psychological

3. Parents

*Corresponding Author: Reza Davarniya

E-mail: rezadavarniya@yahoo.com 
اثربخشى زوج درمانى مبتنى بر تصويرسازى ارتباطى: تأثير آن بر دلزدكى زناشويى

\author{
داود نظريور'، كيانوش زهر اكار '، سيد مصطفى يوريحيى ‘ّ، رضا داورنيا؟" \\ اخروه مشاوره، دانشخاه علامه طباطبايى، تهران، ايران \\ آمروه مشاوره، دانشخاه خوارزمى، تهران، ايران

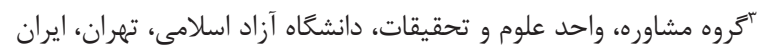

\title{
اطلاعات مقاله:
}

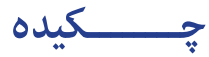

مقدمه: رويكرد تصويرسازى ارتباطى يك نظريه در مورد ازدواج و فرايند درمان تعارضات زناشويى است.

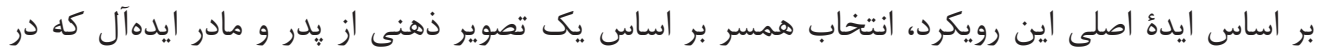

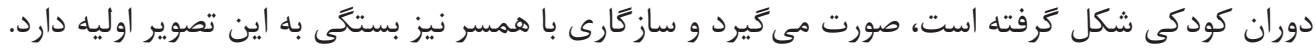

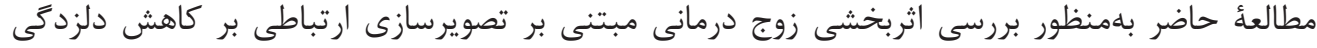

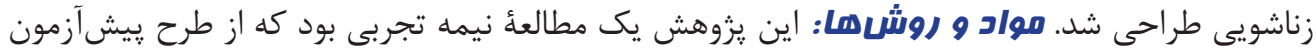

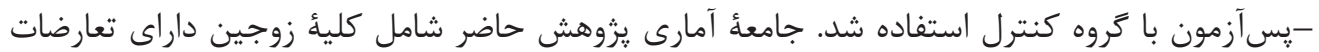

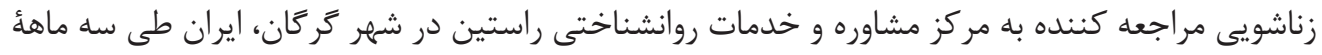

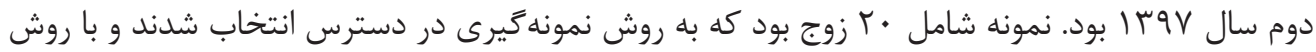

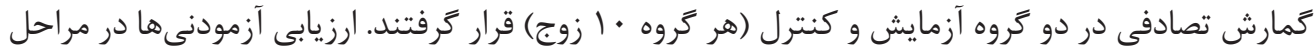

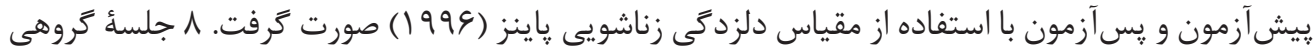



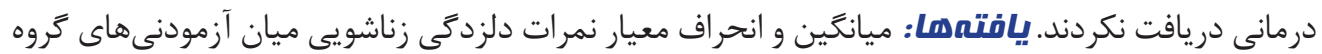

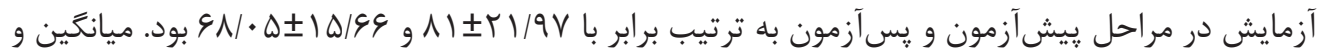

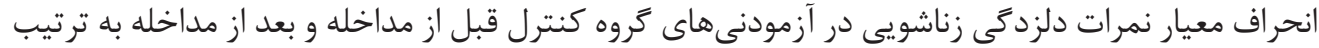

كليد وازهها:

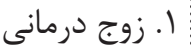
r:

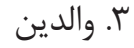

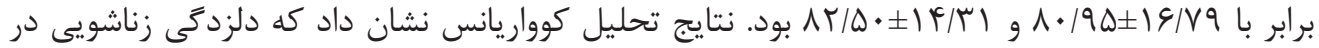

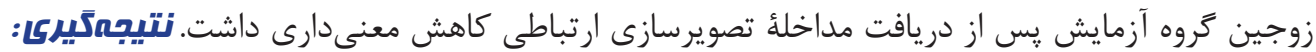

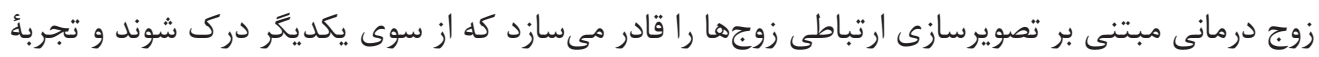

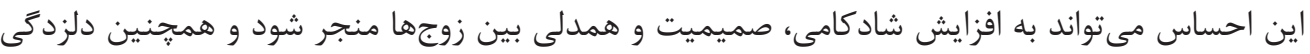
زناشويى را كاهش دهد.

" نويسنده مسئول: رضا داورنيا آدرس الكترونيكى: rezadavarniya@yahoo.com 


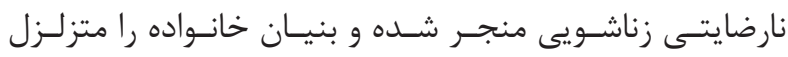

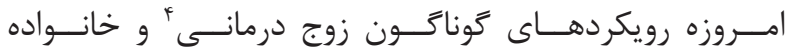

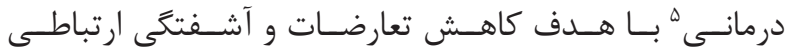

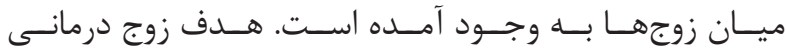

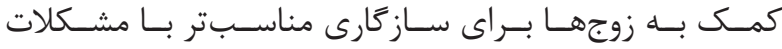


درمـان تصويرسـازى ارتباطـى ؟ يكـى از رويكردهــاى مطــرح

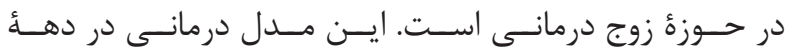

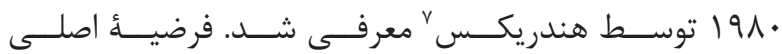

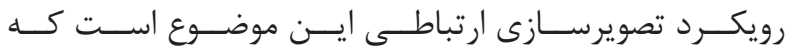

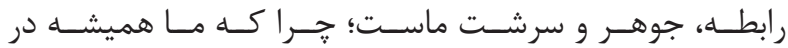



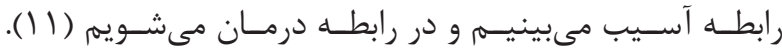

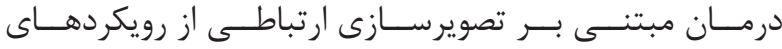

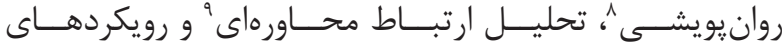



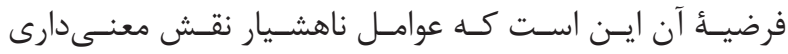

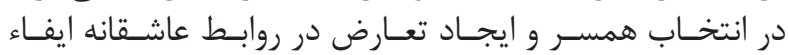

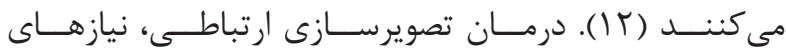

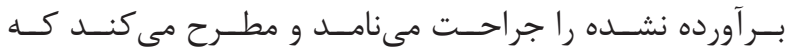

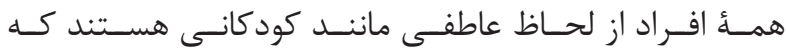



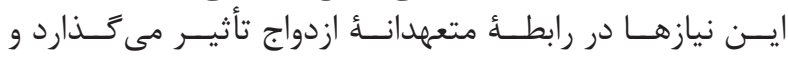



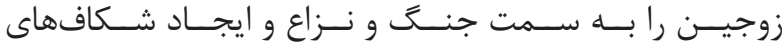

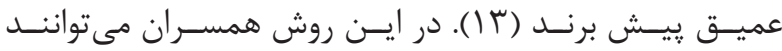

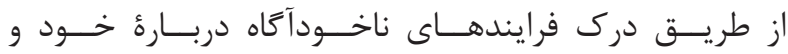

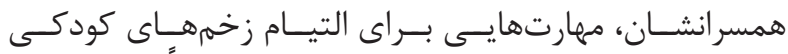

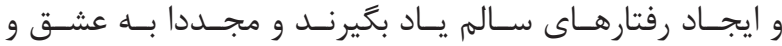

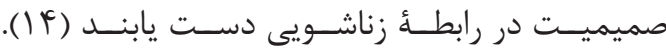



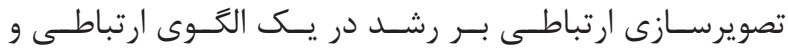

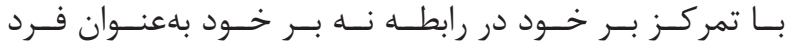

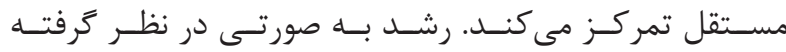

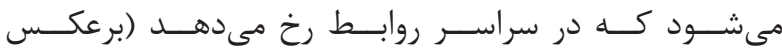



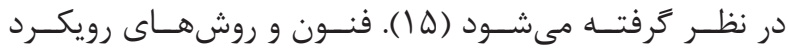

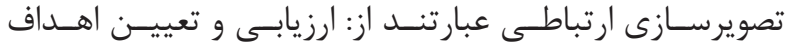

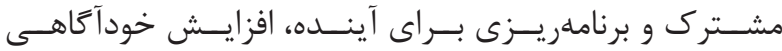

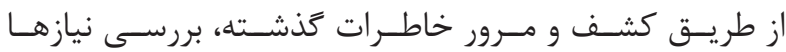

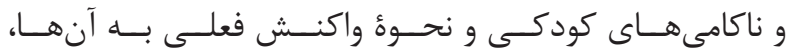

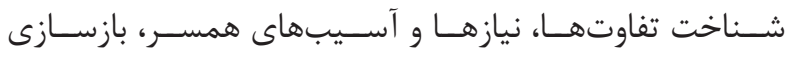

${ }^{1}$ Ellis

${ }^{2}$ Burnout

${ }^{3}$ Pines

${ }^{4}$ Couple therapy

${ }^{5}$ Family therapy

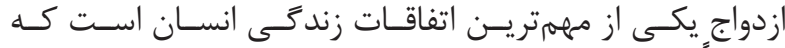

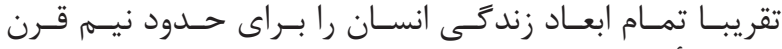

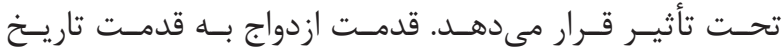

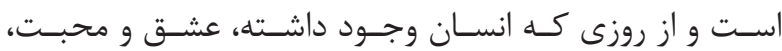

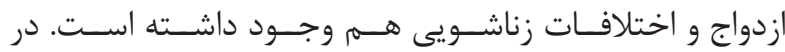

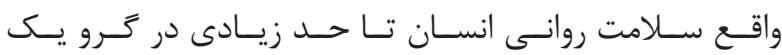

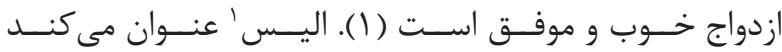

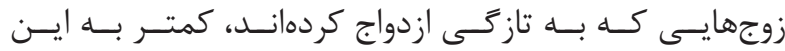



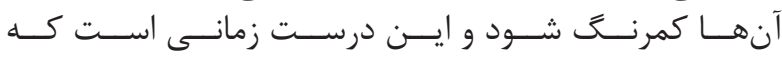

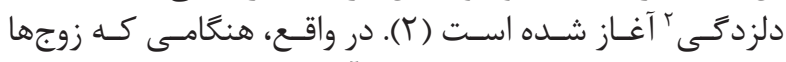

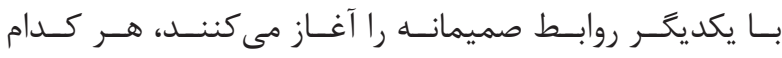

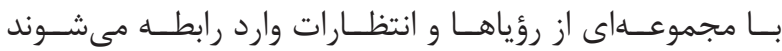

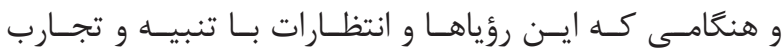

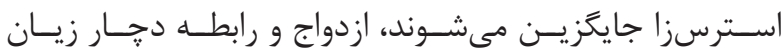

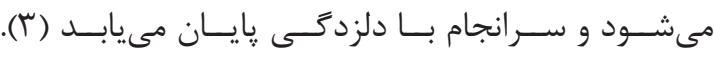
دلزدگَى وضعيـت دردنـاك جسـمانى، عاطفـى و روانسى اسـت

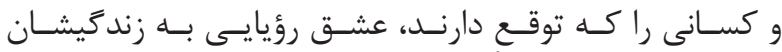

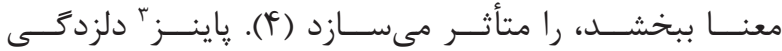

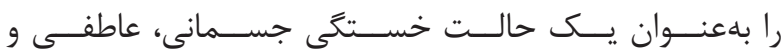

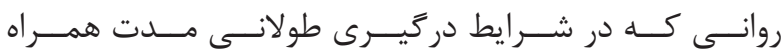

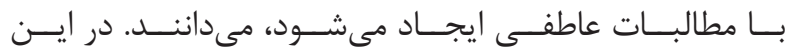

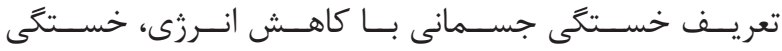

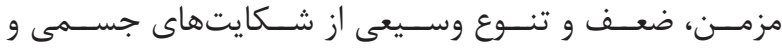

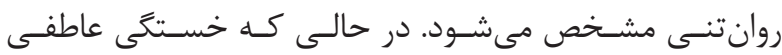

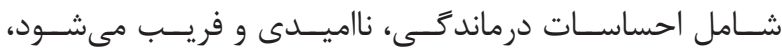

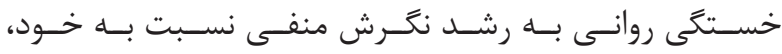

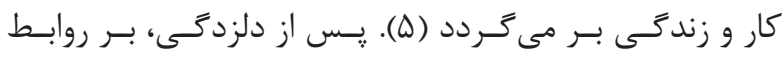

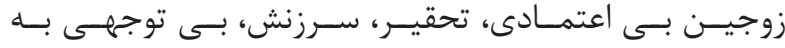

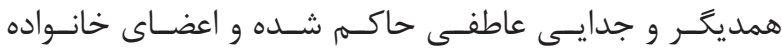

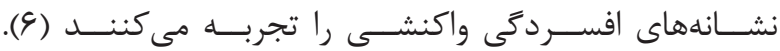

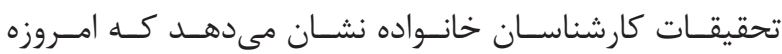

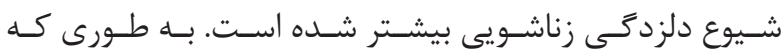

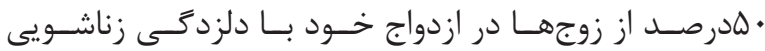

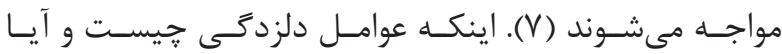

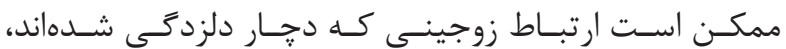

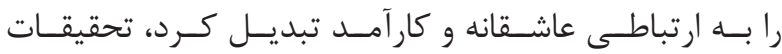



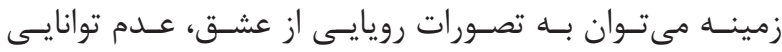

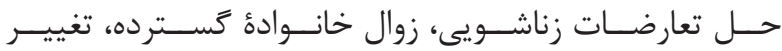

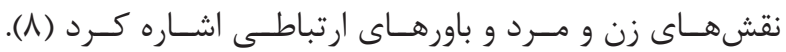

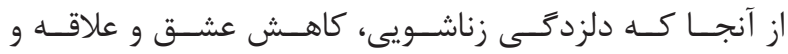

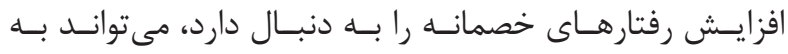

\footnotetext{
${ }^{6}$ Imago relationship therapy

${ }^{7}$ Hendrix

${ }^{8}$ Psychodynamic approaches

${ }^{9}$ Transactional analysis

${ }^{10}$ Cognitive-behavioral approaches
} 


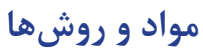

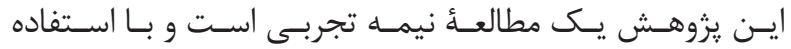

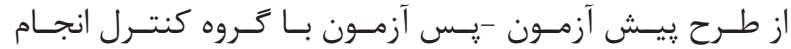

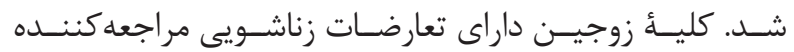

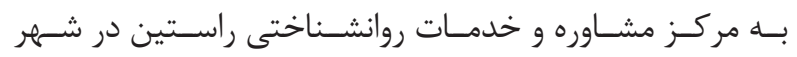

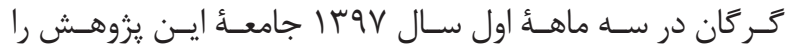



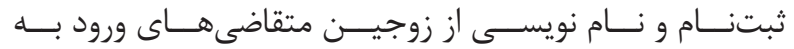

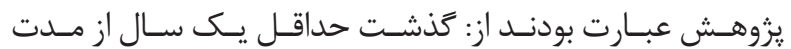

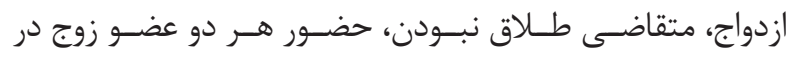



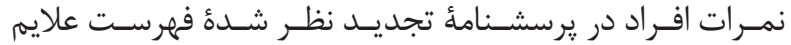

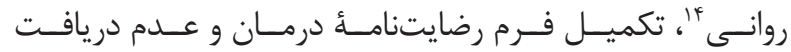

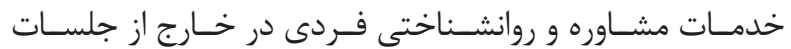

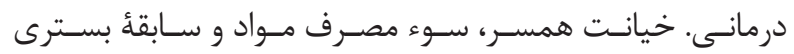

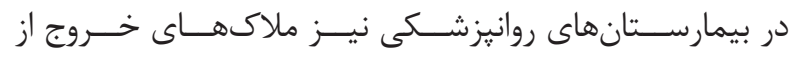

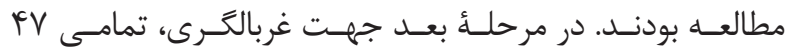

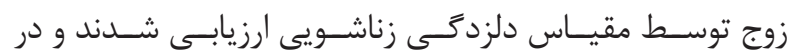

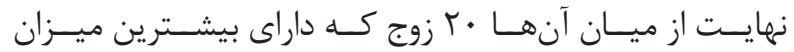

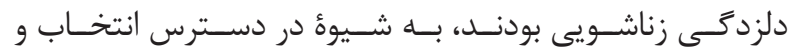

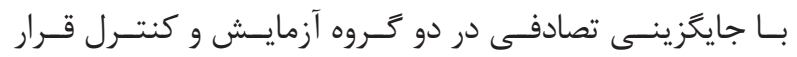

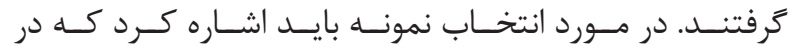

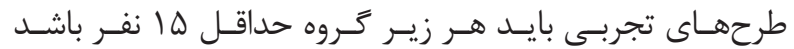

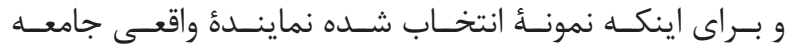

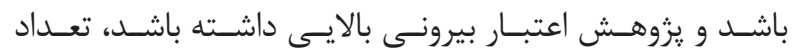

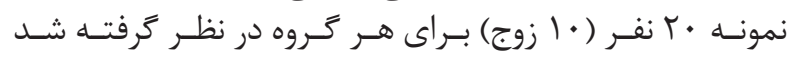

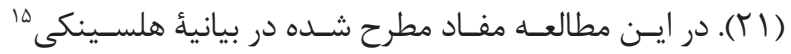

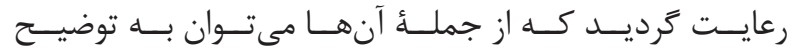

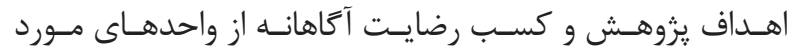

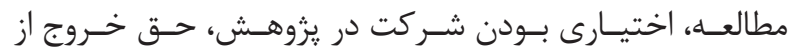

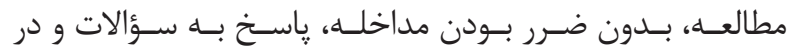

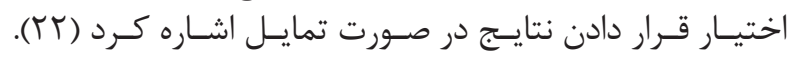

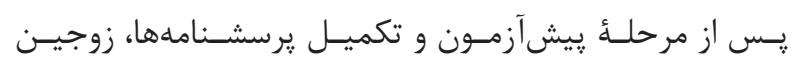

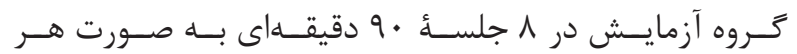

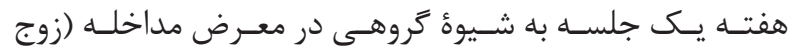

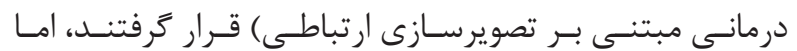

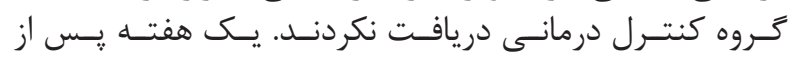

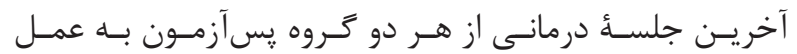

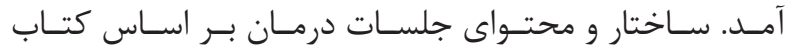

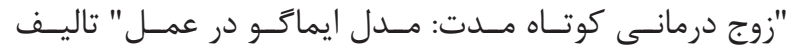

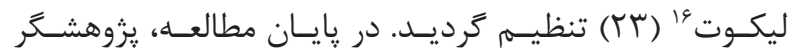

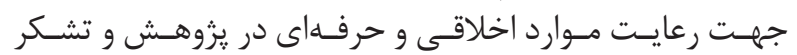

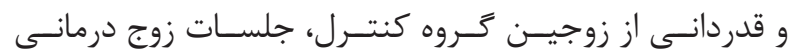

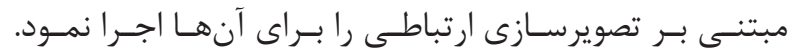

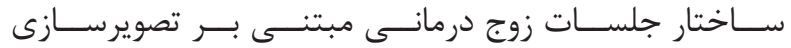

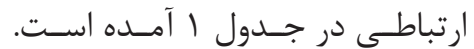

${ }^{11}$ Schmidt, Luquet and Gehlert

${ }^{12}$ Muro, Holliman and Luquet

${ }^{13}$ DeKlerk

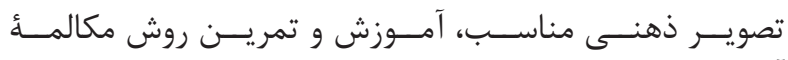

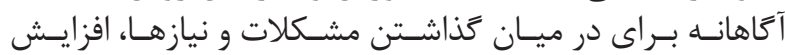

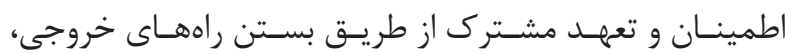

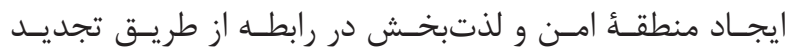

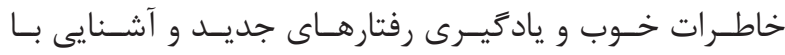



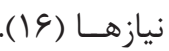

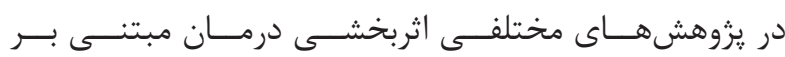

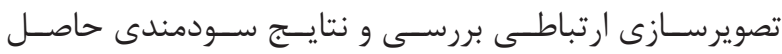



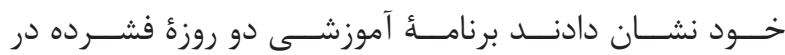

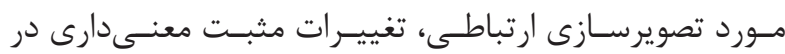

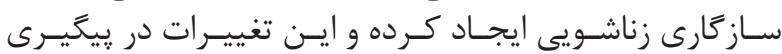

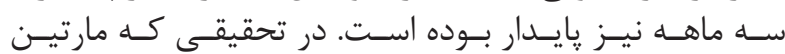

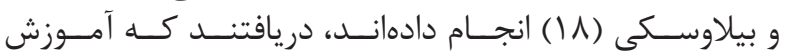

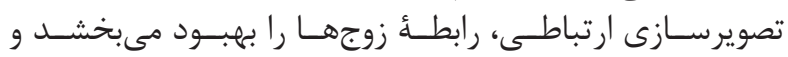

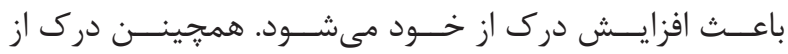

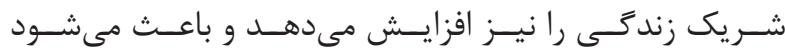

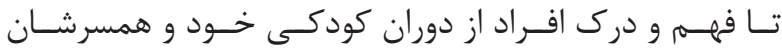

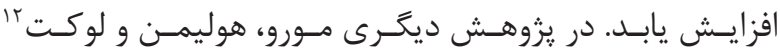

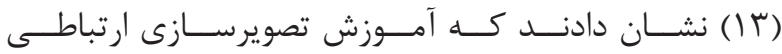

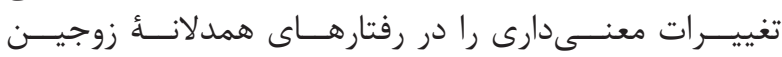

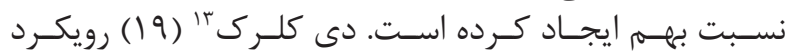

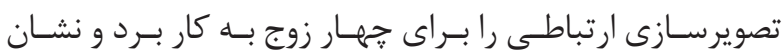

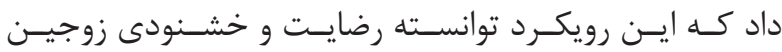

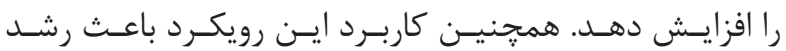

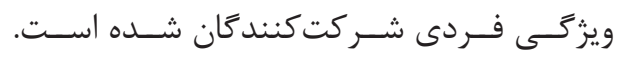

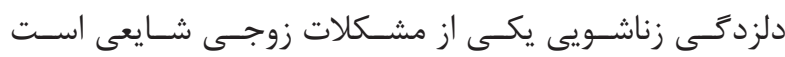

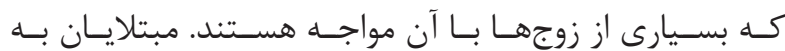

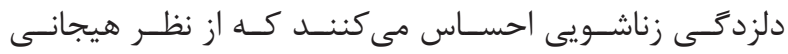

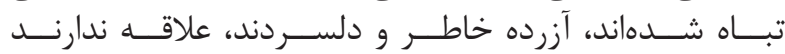

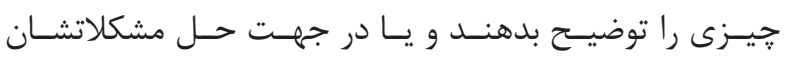

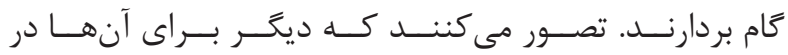

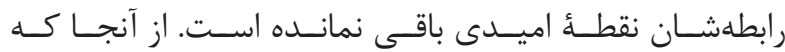

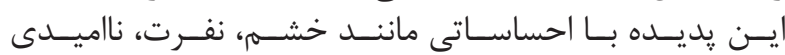

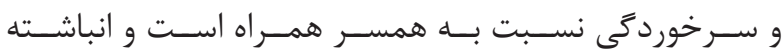

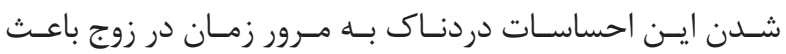

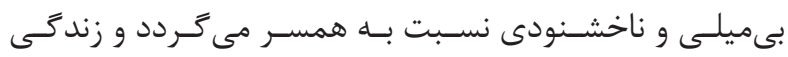

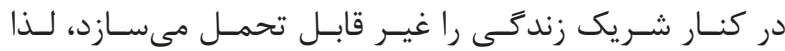

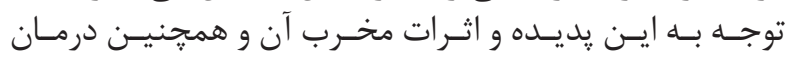

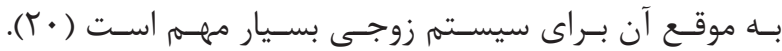

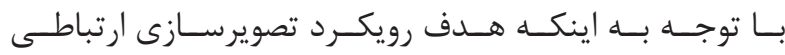

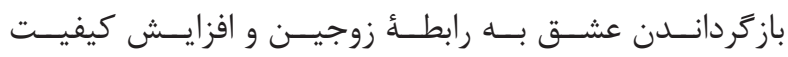

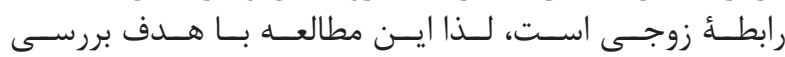

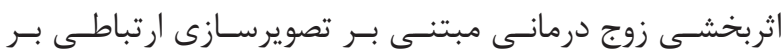

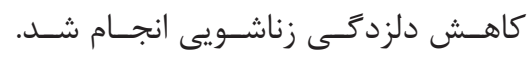

${ }^{14}$ The symptom checklist-90-R (SCL-90-R)

${ }^{15}$ Helsinki

${ }^{16}$ Luquet 


\begin{tabular}{|c|c|c|}
\hline روثى & إمدال & جلسات \\
\hline 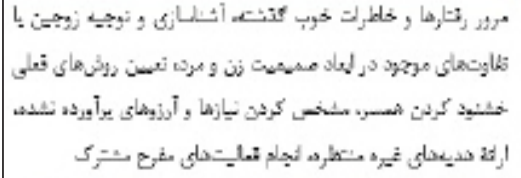 & 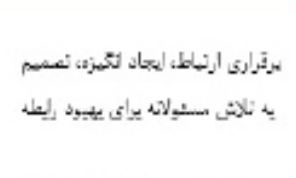 & 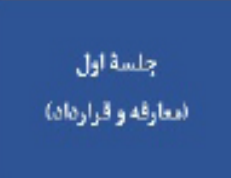 \\
\hline 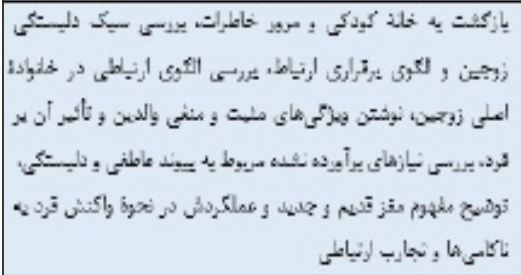 & 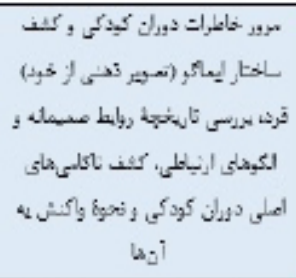 & 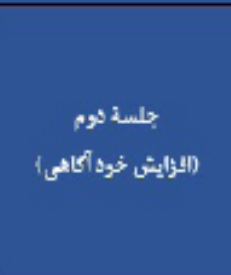 \\
\hline 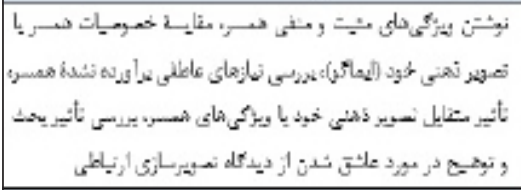 & 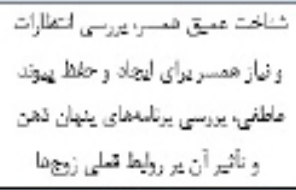 & ) \\
\hline 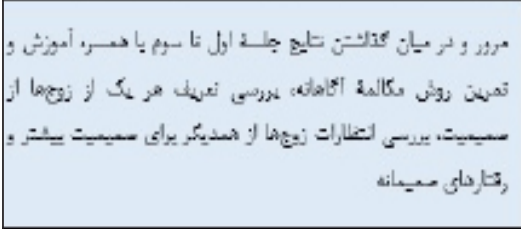 & 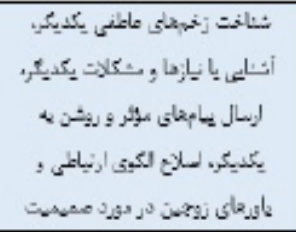 & 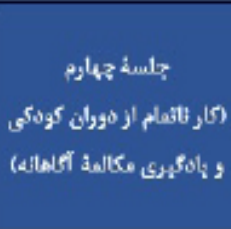 \\
\hline 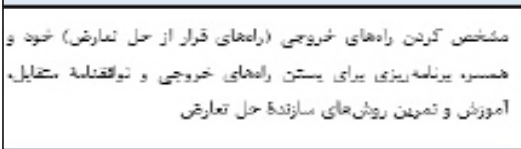 & 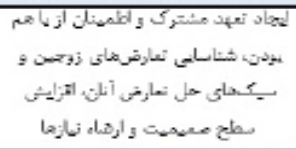 & 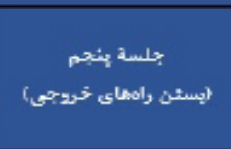 \\
\hline 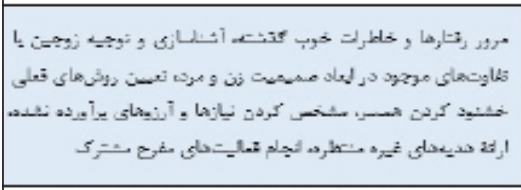 & 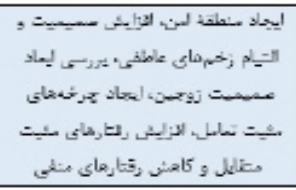 & 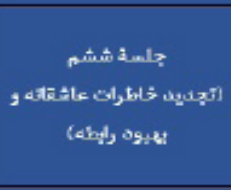 \\
\hline 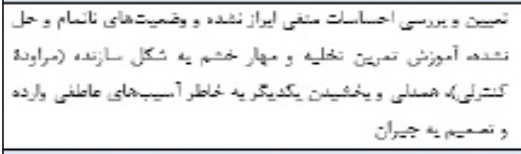 & 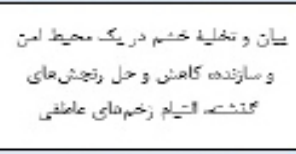 & 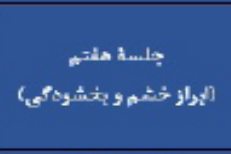 \\
\hline 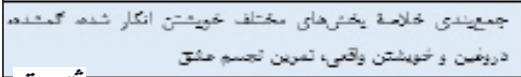 &  & 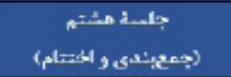 \\
\hline
\end{tabular}

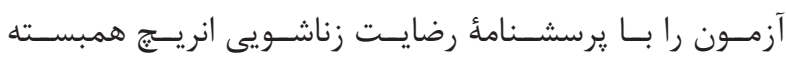

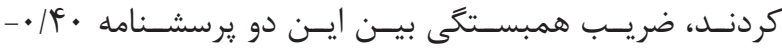

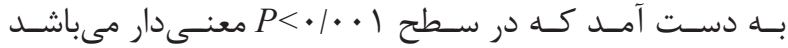

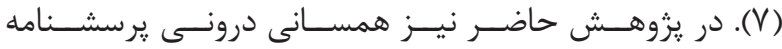

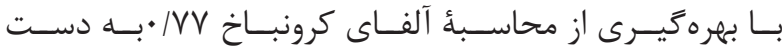

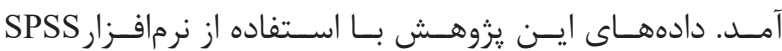

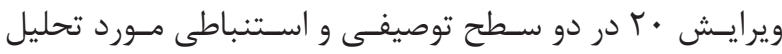

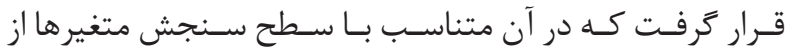

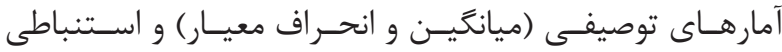

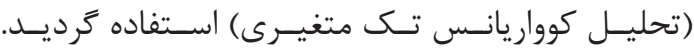

نافته ها

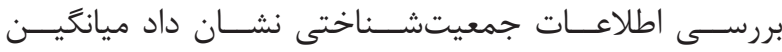


كـروه كنتـرل

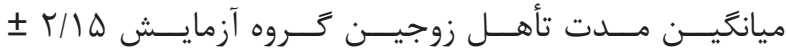
•

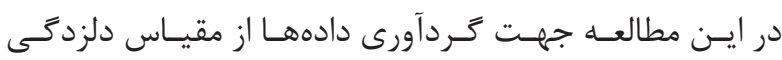

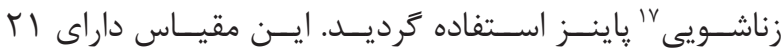



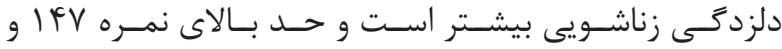

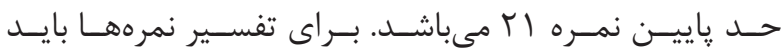

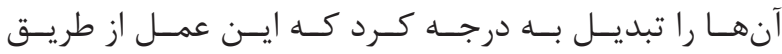

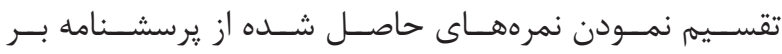

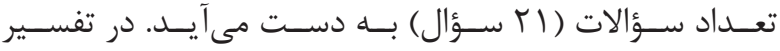

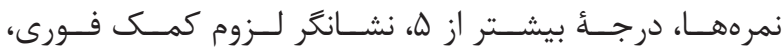

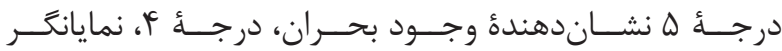

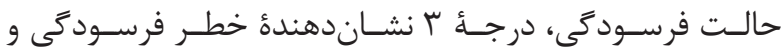

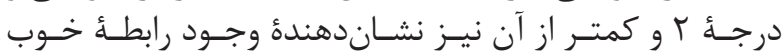

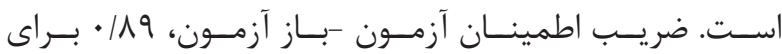

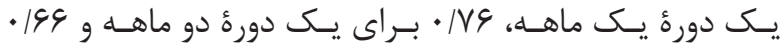

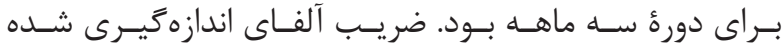

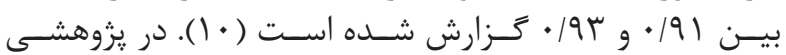

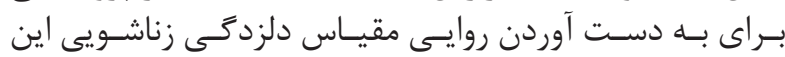

${ }^{17}$ Couple burnout measure 


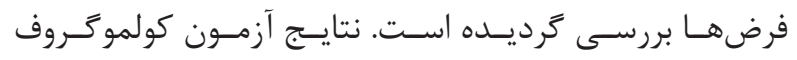
-

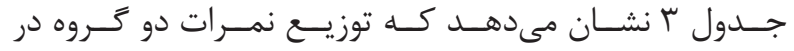

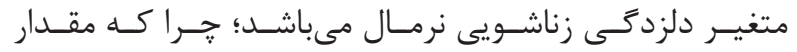

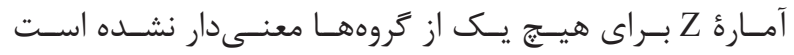

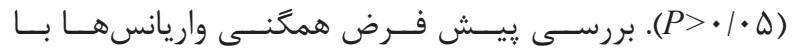

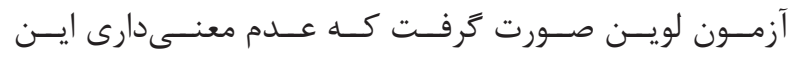

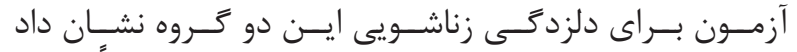

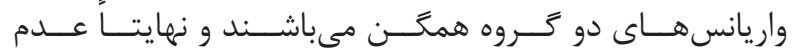

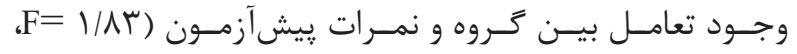

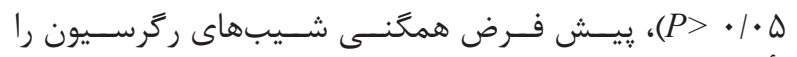
تأييـد كـرد.

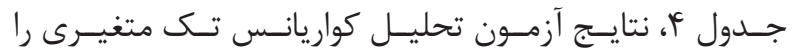



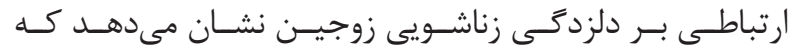

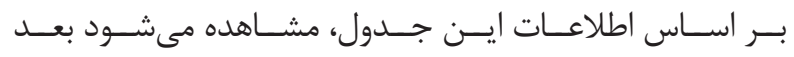

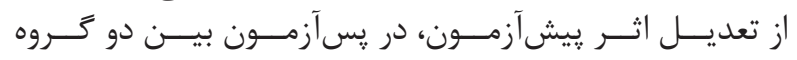

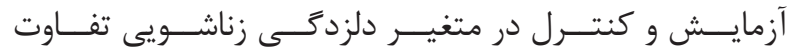

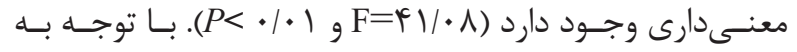

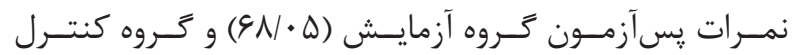

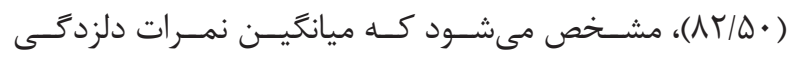

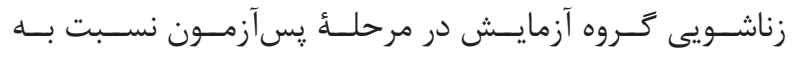

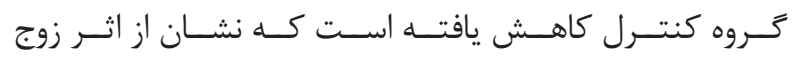

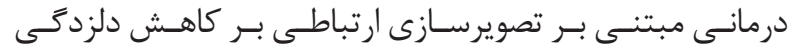

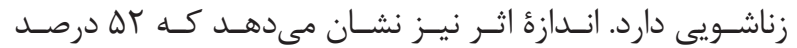

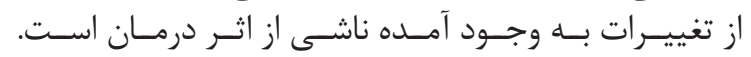



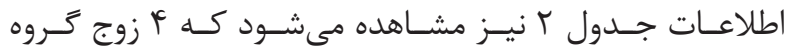

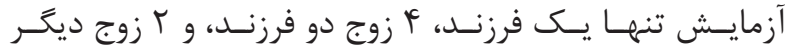

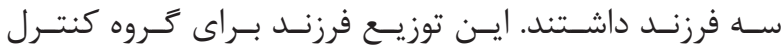

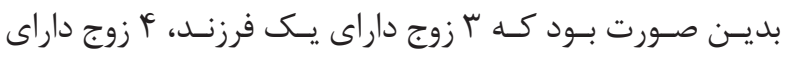

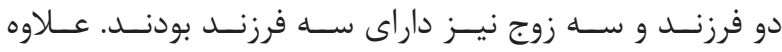

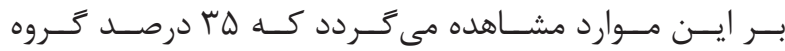

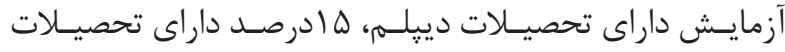

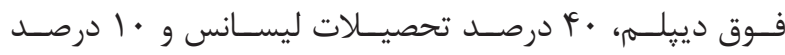

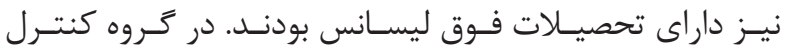

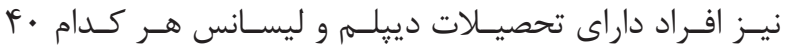



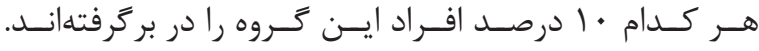

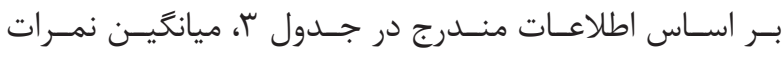

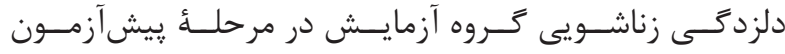

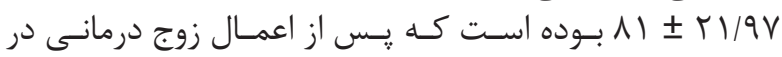

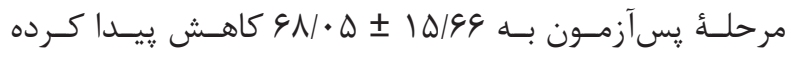

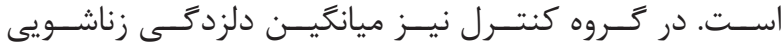

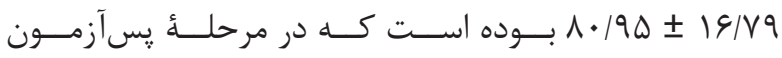

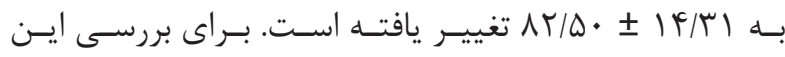

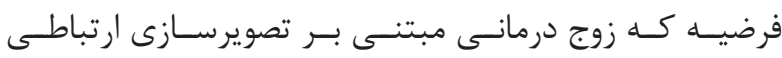

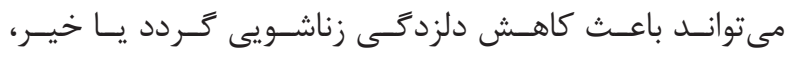

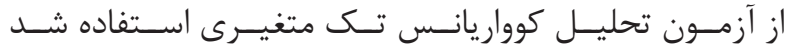

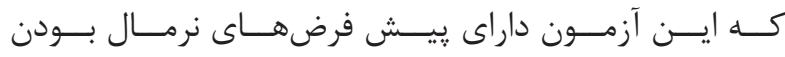

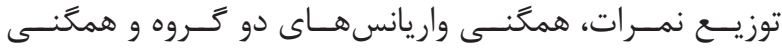

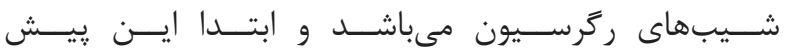

\begin{tabular}{|c|c|c|c|c|c|c|}
\hline درصد & فراوانى & تعداد فرزند & درصد & فراوانى & تحصيلات & كروه \\
\hline$f$. & $f$ & يك فرزند & ra & y & ديبلهم & \multirow{5}{*}{ آزمايش } \\
\hline f. & $f$ & دو فرزند & 10 & $r$ & فوق ديبلم & \\
\hline r. & r & سه فرزند & $f$. & $\wedge$ & ليسانس & \\
\hline 1. & 1. & مجموع & 1. & r & فوق ليسانس & \\
\hline - & - & - & $1 \cdots$ & $r$. & مجموع & \\
\hline$r$. & $r$ & يك فرزند & $f$. & $\wedge$ & ديبلم & \multirow{5}{*}{ كترول } \\
\hline f. & f & دو فرزند & 1. & T & فوق ديِّم & \\
\hline$r$. & $r$ & سه فرزند & f. & $\wedge$ & ليسانس & \\
\hline $1 \cdots$ & 1. & مجموع & 1. & r & فوق ليسانس & \\
\hline - & - & - & $1 \ldots$ & $r$. & مجموع & \\
\hline
\end{tabular}

\begin{tabular}{|c|c|c|c|c|c|}
\hline سطح معنى دارى & مقدار Z & انحراف معيار & ميانكين & \multicolumn{2}{|c|}{ كرؤهo } \\
\hline .1 .99 & $1 / \pi r$ & rI/9Y & 11 & بيش آزمون & كروه \\
\hline . & $\cdot / 9 \cdot r$ & $10 / 99$ & $9 N / \cdot 0$ & يِ آزمون & آزمايش \\
\hline$\cdot \mid A \vee \Lambda$ & $\cdot 1019$ & 19119 & $1 \cdot 190$ & بيش آزمون & كروه \\
\hline . 109. & . / 9. & $|F / \mu|$ & $\Lambda r / \omega$. & يس آزمون & كنترل \\
\hline
\end{tabular}


جدول F- نتايج آزمون تحليل كوواريانس تك متغيرى تأثير زوج درمانى بر دلزدىى زناشويى.

\begin{tabular}{|c|c|c|c|c|c|c|c|}
\hline توان & إندازة اثر & معنى دارى & $\mathrm{F}$ & مجذانكَين & درجهُ آزادى & مجذمورع & متغير \\
\hline - & - & $.1 . \cdot 1$ & $\mid r \cdot / \Delta V$ & $4999 / 19$ & 1 & $9949 / \mathrm{Vq}$ & يَيش آزمون \\
\hline 1 & - $/ \Delta T$ & $.1 . \cdot 1$ & $\mid+1 / \cdot 1$ & T. QV/AT & 1 & T. QV/AT & زوج درمانى \\
\hline & & & & $\Delta 1 / \cdot 0$ & rV & $1419 / 10$ & خطا \\
\hline & & & & & r. & rrvrqy & مجموع \\
\hline
\end{tabular}

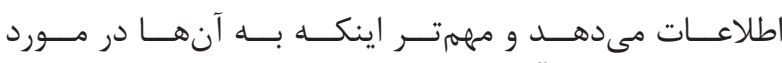

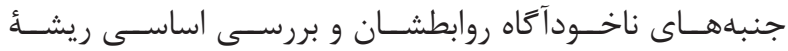

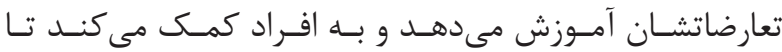

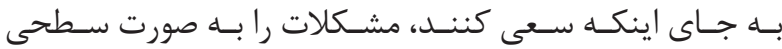



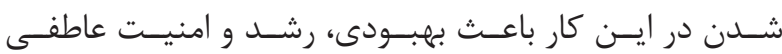

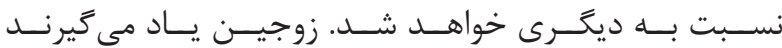

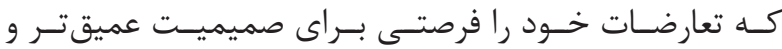

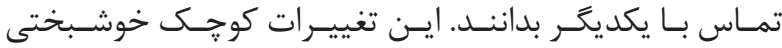

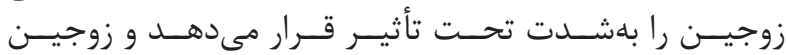

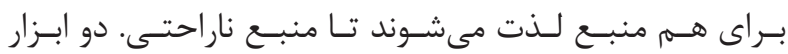

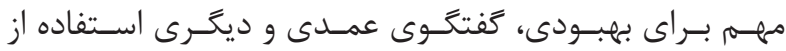

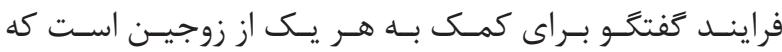

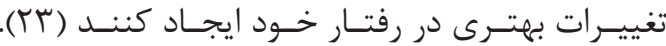

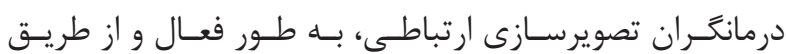

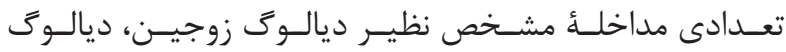



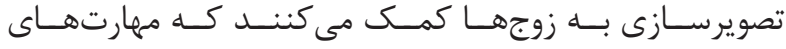





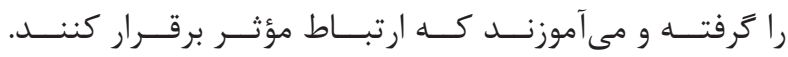

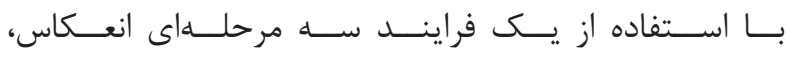

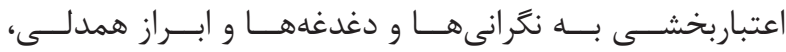

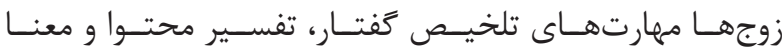

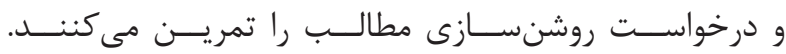



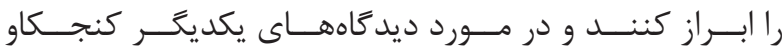

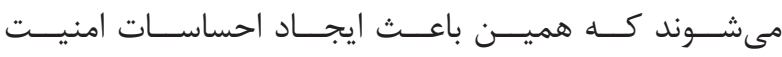

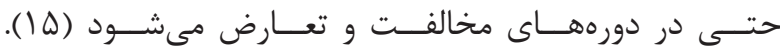

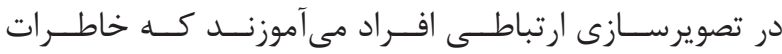

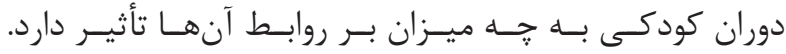

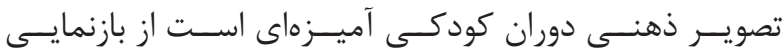

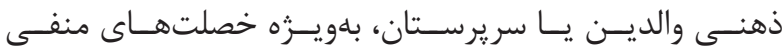

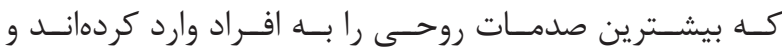

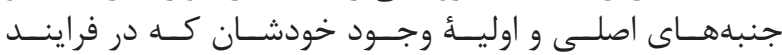

${ }^{18}$ Muro and Holliman

${ }^{19} \mathrm{Schmidt}$

${ }^{20}$ DeKlerk
بحث و نتيجهَ

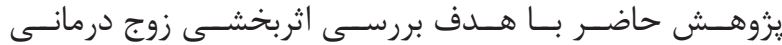

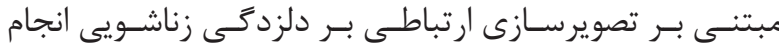



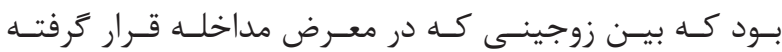

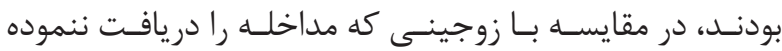

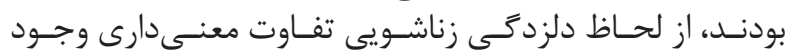

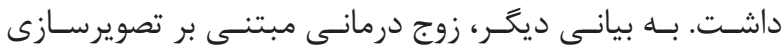

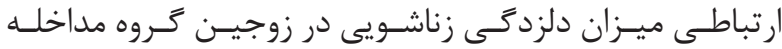

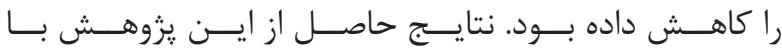



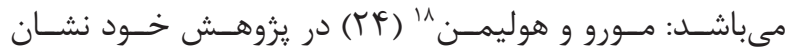

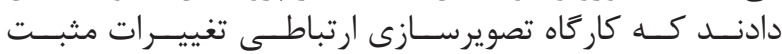

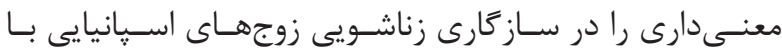

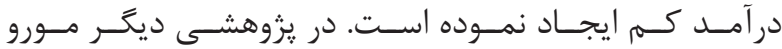

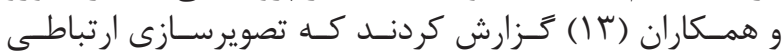

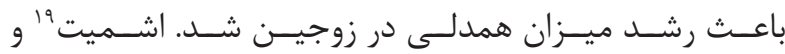

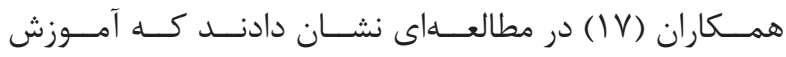

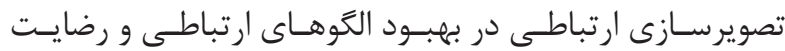

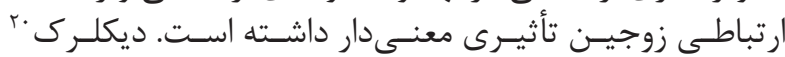

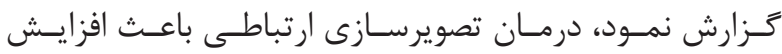

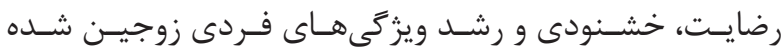

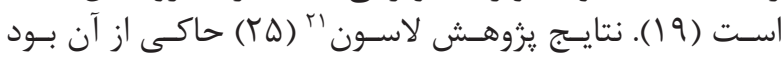

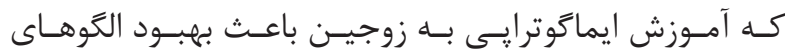

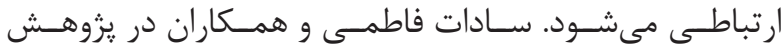

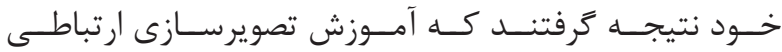

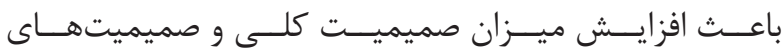

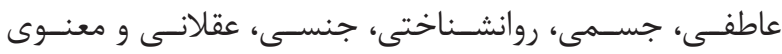

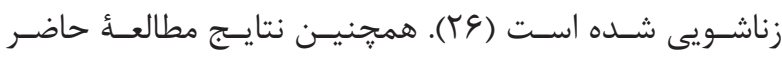

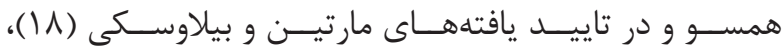

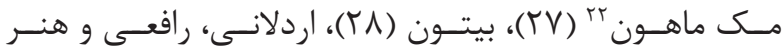

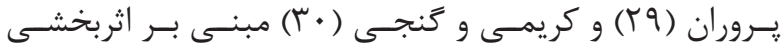

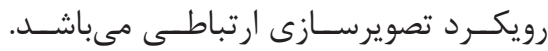

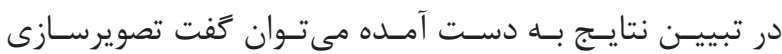

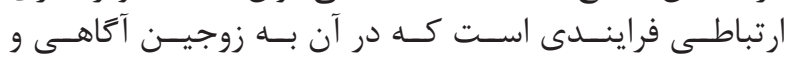

${ }^{21}$ Lawson

${ }^{22}$ McMahon 


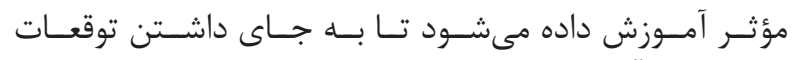

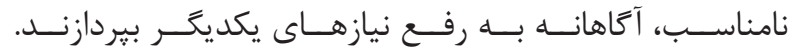

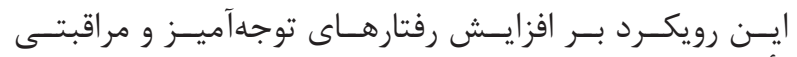

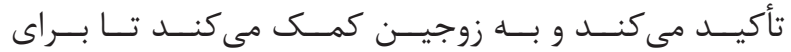

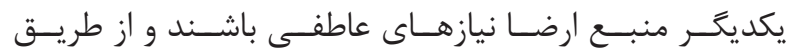



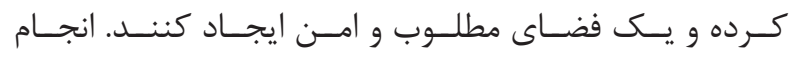

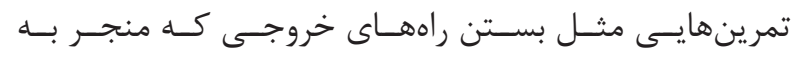

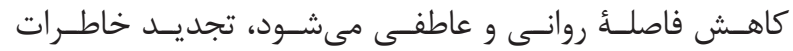

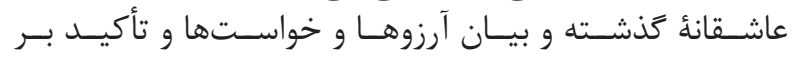

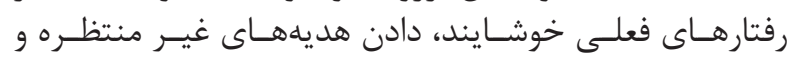

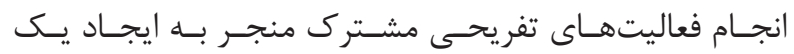

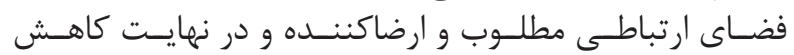

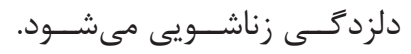

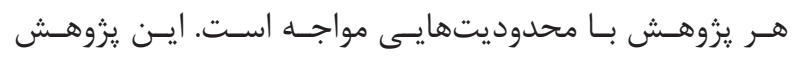



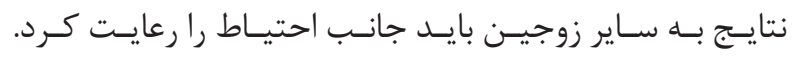

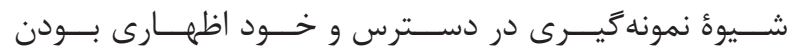

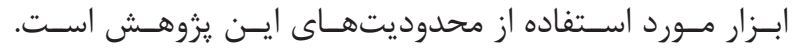

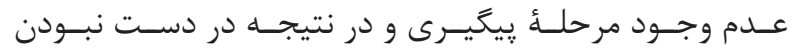

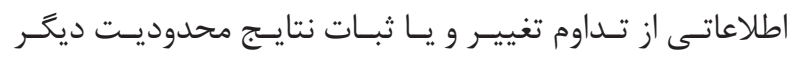

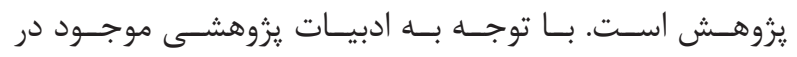

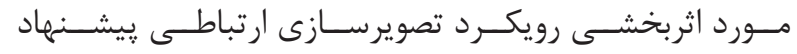

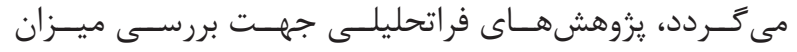

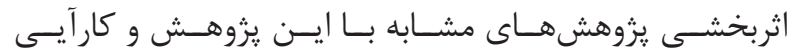

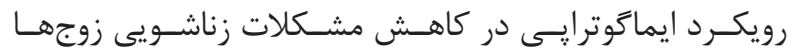

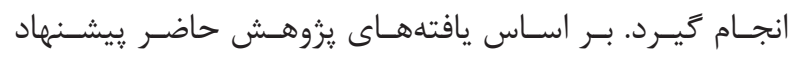

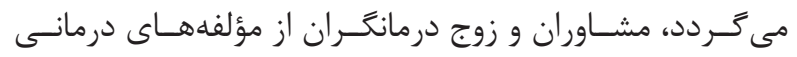

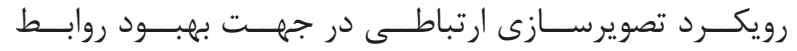

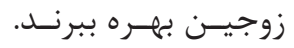

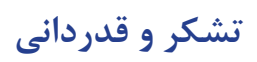

حَـروه يزوهشـى مراتـب تقديسر و تشـكر خـود را از مديريست

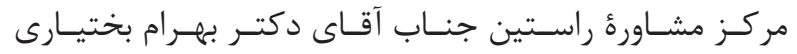

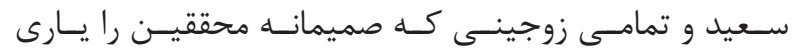

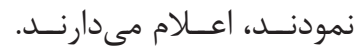

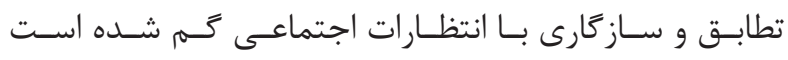

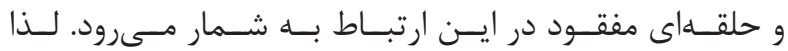

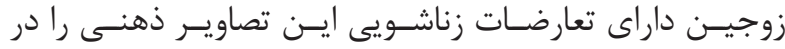

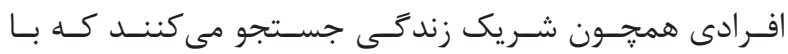

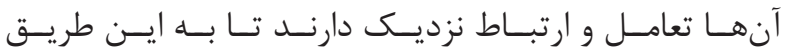

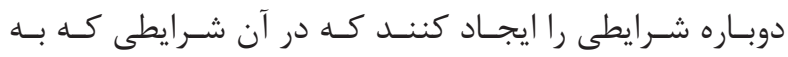



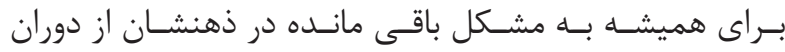

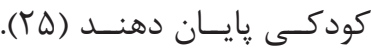

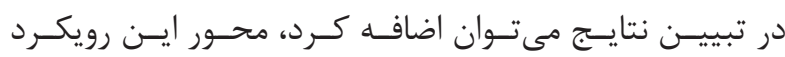



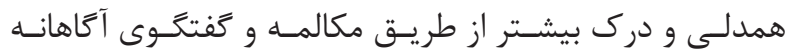

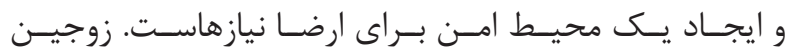

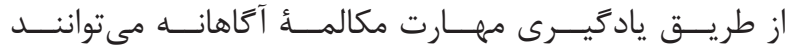

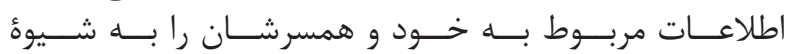

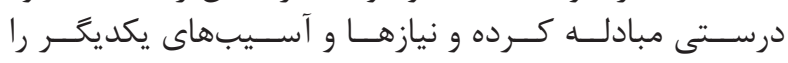

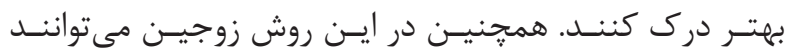

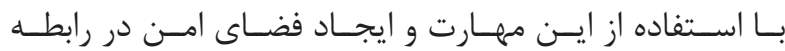

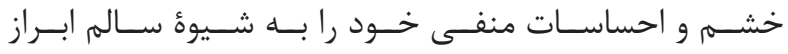

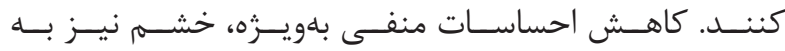

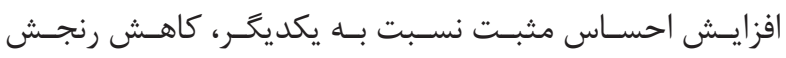

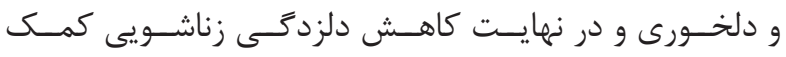

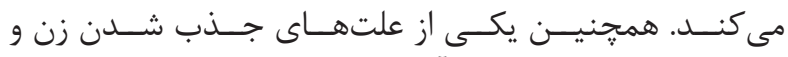

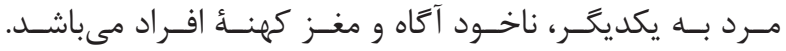

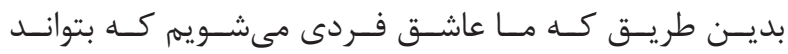

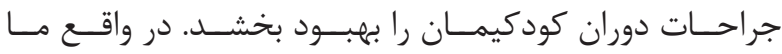

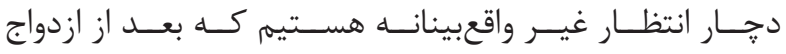

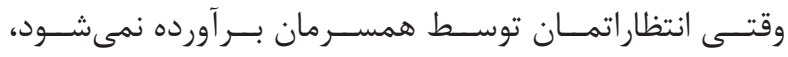

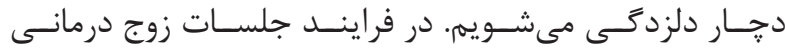

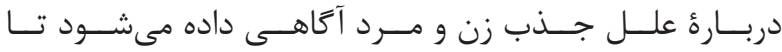



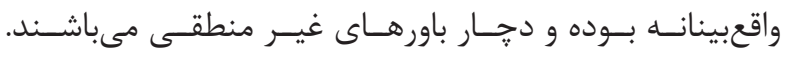

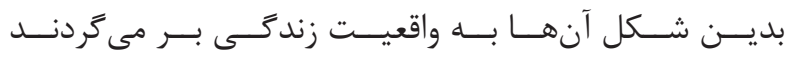

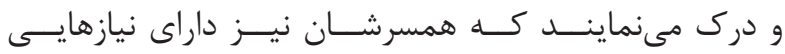

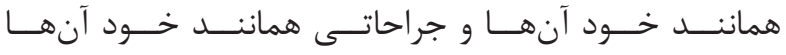

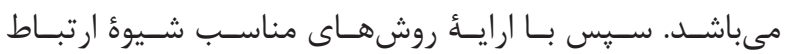


1. HosseiniA, Rasouli M, Davarniya R, Babaeigarmkhani M. The effect of group training based on practical application of intimate relationship skills program on marital relationships quality. JIUMS. 2016; 24(2): 8-17.

2. Koolaee AK, Adibrad N, Sedgh Poor BS. The comparison of relationship beliefs and couples burnout in women who apply for divorce and women who want to continue their marital life. Iran J Psychiatry. 2010; $5(1): 35-9$

3. Pines AM. Adult attachment styles and their relationship to burnout: a preliminary and crosscultural investigation. An International Journal of Work, Health and Organisations. 2004; 18(1):66-80.

4. Ahmadi A, Zahrakar K, Davarniya R, Rezaiee M. The effectiveness of brief self-regulation couple therapy on couple burnout in couples of Saveh city. Razi Journal of Medical Sciences. 2015; 22(139): 64-75.

5. Pines AM, Neal MB, Hammer LB, Icekson T. Job burnout and couple burnout in dual- earner couples in the sandwiched generation. Social Psychology Quarterly. 2011; 74 (4):361-86.

6. Asgari A, Goodarzi K. The effectiveness of emotional schema therapy on marital burnout on the brink of divorce. Middle Eastern Journal of Disability Studies. 2018; 8(55):1-9.

7. Sanai B, Davarniya R, Bakhtiari Said B, Shakarami M. The effectiveness of solution-focused brief therapy (SFBT) on reducing couple burnout and improvement of the quality of life of married women. Armaghane Danesh. 2015; 20 (5):416-32.

8. Nazari AM, Rasouli M, Davarniya R, Hosseini A, Babaei Gharmkhani M. Effectiveness of solutionfocused brief therapy (SFBT) on couple burnout and divorce tendency in married women. Iranian Journal of Psychiatric Nursing. 2015; 3(11):41-52.

9. Huston T. What is love got to do with it? Why some marriage succeed and other fail. Journal of Personal Relationship. 2009; 16(3): 301-27.

10. Mohammadi M, Sheykh Hadi Siruii R, Garafar A, Zahrakar K, Shakarami M, Davarniya R. Effect of group cognitive behavioral couples therapy on couple burnout and divorce tendency in couples. Armaghane Danesh. 2017; 21(11): 1069-86.

11. Flemke KR, Protinsky H. Imago dialogues: treatment enhancement with EMDR. Journal of Family Psychotherapy. 2001; 12(4): 1-14.

12. Zielinski JJ. Discovering imago relationship therapy. Psychotherapy: Theory, Research, Practice, Training. 1999; 36(1): 91-101.

13. Muro L, Holliman R, Luquet W. Imago relationship therapy and accurate empathy development. Journal of Couple \& Relationship Therapy. 2015; 15(3): 232-46.

14. Alipour S, Ezazi Bojnourdi E, Honarmand Dorbadam M, Moosavi S, Dasht Bozorgi Z. Effectiveness of imago therapy training on perspective taking and forgiveness in females damaged by marital infidelity. JHPM. 2018; 6(6): 46-52.

15. Gehlert NC. Randomized controlled trial of imago relationship therapy: exploring statistical and clinical significance. (dissertation). Journal of Couple \& Relationship Therapy. 2017; 16(3): 188-209.

16. Robbins CA. ADHD couple and family relationships: enhancing communication and understanding through imago relationship therapy. J Clin Psychol. 2005; 61(5): 565-77.

17. Schmidt CD, Luquet W, Gehlert NC. Evaluating the Impact of the "Getting the love you want" couples workshop on relational satisfaction and communication patterns. Journal of Couple \& Relationship Therapy. 2015; 15(1): 1-18.

18. Martin TL, Bielawski, DM. What is the African American's experience following Imago education? Journal of Humanistic Psychology. 2011; 51(2): 216-28.

19. DeKlerk JH. Imago relationship therapy and christian marriage counseling. (dissertation). Ranp Afrikaans University. 2001; P. 12-13.

20. Morshedi M, Davarniya R, Zahrakar K, Mahmudi MJ, Shakarami M. The effectiveness of acceptance and commitment therapy (ACT) on reducing couple burnout of couples. IJNR. 2015; 10(4): 76-87.

21. Farahani HA, Arizi HR. Translated: methods in behavioral sciences. 1th ed. Tehran: Nashr Ravan Press. 2008; P. 77-8.

22. World Medical Association. World medical association declaration of helsinki: ethical principles for medical research involving human subjects. Bull World Health Organ. 2001; 79(4): 373-4 
23. Luquet W. Short-term couples therapy-The imago model in action. London: Routledge Publication. 2007; P. 150-60.

24. Muro L, Holliman R. Relationship workshop with high ‘risk, hispanic couples. North Carolina Perspectives. 2014; 9(1): 51-62.

25. Lawson W. Improving couple communication through the imago getting the love you want workshop for couples. Stellenbosch University. 2008; P. 95-8.

26. Sadat Fatemi M, Karbalai A, Meygoni M, Kakavand A. The effect of imago therapy on marital intimacy among couples. Knowledge and Research in Applied Psychology. 2015; 17(1): 51-9.

27. McMahon MN. Applying Stolorow's theory of intersubjectivity to Hendrix's imago techniques. Smith College Studies in Social Work. 1999; 69(2): 309-34.

28. Beeton TA. Dyadic adjustment and use of imago skills past participants of the "getting the love you want" workshop for couples. Unpublished Doctoral Dissertation.Walden University; 2005.

29. Ardalani L, Refahi Z, Honarparvaran N. Evaluate the effectiveness of training imago-therapy on willingness to forgive in betrayed couples. Indian Journal of Positive Psychology. 2016; 7(1): 88-92.

30. Karimi E, Ganji K. The effectiveness of imago relationship therapy on women's mental health and resiliency in health centers of Tehran, district 4. Asian Journal of Research in Social Sciences and Humanities. 2016; 6(4): 685-94. 